\title{
Generation and tumor recognition properties of two human monoclonal antibodies specific to cell surface anionic phospholipids
}

\author{
Emil Bujak ${ }^{1} \cdot$ Francesca Pretto $^{2} \cdot$ Dario Neri $^{1}$
}

Received: 24 March 2015 / Accepted: 29 April 2015 /Published online: 19 May 2015

(C) Springer Science+Business Media New York 2015

\begin{abstract}
Summary Phosphatidylserine (PS) and other anionic phospholipids, which become exposed on the surface of proliferating endothelial cells, tumor cells and certain leukocytes, have been used as targets for the development of clinicalstage biopharmaceuticals. One of these products (bavituximab) is currently being investigated in Phase 3 clinical trials. There are conflicting reports on the ability of bavituximab and other antibodies to recognize PS directly or through beta-2 glycoprotein 1, a serum protein that is not highly conserved across species. Here, we report on the generation and characterization of two fully human antibodies directed against phosphatidylserine. One of these antibodies (PS72) bound specifically to phosphatidylserine and to phosphatidic acid, but did not recognize other closely related phospholipids, while the other antibody (PS41) also bound to cardiolipin. Both PS72 and PS41 stained 8/9 experimental tumor models in vitro, but both antibodies failed to exhibit a preferential tumor accumulation in vivo, as revealed by quantitative biodistribution analysis. Our findings indicate that anionic phospholipids are exposed and accessible in most tumor types, but cast doubts about the possibility of efficiently targeting tumors in vivo with PS-specific reagents.
\end{abstract}

Electronic supplementary material The online version of this article (doi:10.1007/s10637-015-0248-0) contains supplementary material, which is available to authorized users.

Dario Neri

neri@pharma.ethz.ch

1 Institute of Pharmaceutical Sciences, Department of Chemistry and Applied Biosciences, ETH Zürich, Vladimir-Prelog-Weg 2, CH-8093 Zurich, Switzerland

2 Philochem AG, Libernstrasse 3, CH-8112 Otelfingen, Switzerland
Keywords Phosphatidylserine $\cdot$ Phage display $\cdot$ Antibody · Small immune protein (SIP) $\cdot$ Pharmacodelivery $\cdot$ Tumor targeting

\begin{tabular}{|c|c|}
\hline \multicolumn{2}{|c|}{ Abbreviations } \\
\hline PS & Phosphatidylserine \\
\hline$\beta 2 \mathrm{GP} 1$ & Beta-2 glycoprotein 1 \\
\hline DPPS & $\begin{array}{l}\text { 1,2-Dipalmitoyl-sn-glycero-3-phosphoserine, sodi- } \\
\text { um salt }\end{array}$ \\
\hline DPPA & $\begin{array}{l}\text { 1,2-Dipalmitoyl-sn-glycero-3-phosphate, sodium } \\
\text { salt }\end{array}$ \\
\hline DPPC & 1,2-Dipalmitoyl-sn-glycero-3-phosphocholine \\
\hline DPPE & $\begin{array}{l}\text { 1,2-Dipalmitoyl-sn-glycero-3- } \\
\text { phosphoethanolamine }\end{array}$ \\
\hline SM & Sphingomyelin \\
\hline CL & Cardiolipin \\
\hline PI & Phosphatidylinositol \\
\hline
\end{tabular}

\section{Introduction}

Phosphatidylserine (PS) is the main anionic phospholipid constituent of cellular membranes and is almost exclusively located in the inner leaflet of the plasma membrane. Loss of phospholipid asymmetry, demonstrated mainly by migration of PS to the outer leaflet of cell membrane, is a hallmark of apoptosis. In fact, PS exposure is required for phagocytosis of apoptotic cells by macrophages and fibroblasts [1]. Apoptosis-related PS exposure is independent of the trigger of apoptosis and of cell type.

Surface exposure of PS has been reported to be a common feature for the plasma membrane of cancer cells, tumor endothelial cells and certain leukocytes [2-5], while this event appears to be rare in healthy tissues [6]. These claims were based on staining experiments, performed both with Annexin V 
reagents and with monoclonal antibodies. As a consequence, protein-based biopharmaceuticals, capable of selective PS recognition, have been proposed as tools for vascular tumor targeting and for imaging applications in vivo [7-13]. In addition, the antibody-based blockade of PS has been reported to increase tumor-derived antigen presentation by dendritic cells and the generation of tumor-specific T cells $[14,15]$.

The mouse monoclonal antibody $3 \mathrm{G} 4$ and its chimeric antibody derivative (bavituximab) were initially claimed to directly recognize PS on tumor endothelial cells, to target tumor blood vessels in vivo and to potently inhibit cancer growth in tumorbearing mice [16-18, 15]. After the initial report of direct PS recognition, 3G4 and bavituximab were shown to recognize beta-2 glycoprotein 1 (" $\beta 2 \mathrm{GP} 1$ ", a low-affinity PS binder) and, thus, to recognize PS only indirectly. According to the proposed mechanism, the $3 \mathrm{G} 4$ antibody would recognize PS in the presence of human $\beta 2 \mathrm{GP} 1$, but not in the presence of the mouse analogue of the protein [19]. This finding is not surprising, considering the fact that human and murine $\beta 2 \mathrm{GP} 1$ share only $76 \%$ identity [20]. However, according to previous reports, the $3 \mathrm{G} 4$ antibody would be able to selectively localize to tumor blood vessels in mice, without the injection of human $\beta 2 \mathrm{GP1}$ [19]. To the best of our knowledge, quantitative biodistribution studies in tumor-bearing mice with $3 \mathrm{G} 4$ or bavituximab have not been published.

More recently, Stafford et al. reported the isolation and characterization of phage-derived human antibodies, which directly bind to human $\beta 2 \mathrm{GP} 1$ and which allegedly selectively localize to prostate tumors in mice [21]. A close inspection of the experimental data, however, reveals that the tumor:blood ratios for this antibody were low (1:1 $48 \mathrm{~h}$ after intravenous administration) and similar to the ones observed for an antibody of irrelevant specificity in the mouse (used as negative control), which, however, exhibited a strikingly faster clearance. The negative control antibody, which was used in $\mathrm{F}(\mathrm{ab})_{2}$ format, appeared to clear faster compared to other $\mathrm{F}\left(\mathrm{ab} \mathrm{b}_{2}\right)_{2}$ antibodies for which quantitative biodistribution studies have been published [22].

In this article, we report on generation and characterization of two monoclonal antibodies (PS41 and PS72) that directly recognize phosphatidylserine in the absence of $\beta 2 \mathrm{GP} 1$. The two antibodies strongly stained the majority of the experimental tumor models tested, with a staining pattern that was vascular for certain tumors (e.g., U87) or more diffuse for other neoplasms (e.g., HeLa, SKRC-52). Importantly, no staining was detectable in healthy adult mouse organ sections.

\section{Results}

\section{Generation and characterization of anti-PS antibodies}

A large synthetic phage display library, containing $>40$ billion human antibody clones [23], was used for the selection of antibodies in a scFv format, specific to PS. 1,2-dipalmitoylsn-glycero-3-phosphoserine, sodium salt (DPPS) (Fig. 1a) was immobilized on Maxisorp plastic plates and binding clones were isolated after 3 rounds of panning. The two antibodies that gave the strongest signal in ELISA (PS41 and PS72) were converted into small immune protein (SIP) format [24, 25] (Fig. 1b), cloned and expressed in mammalian cells. The antibody sequences differ at the level of the complementarity-determining region (CDR) 3 loops, which were the combinatorially mutated regions in the parental phage display library (Fig. 1c).

SIP(PS41) and SIP(PS72) were purified to homogeneity using affinity chromatography procedures. The antibodies bound to DPPS-coated microsensor chips in a BIAcore analysis (Fig. 2). Monomeric preparations of $\mathrm{scFv}(\mathrm{PS} 41)$ and $\mathrm{scFv}(\mathrm{PS} 72)$, obtained by size exclusion chromatography, bound to the same chip, revealing a $\mathrm{Kd}$ of $65 \mathrm{nM}$ for PS41 and 25nM for PS72 [Supplementary Figure 1]. As a control for binding studies, SIP(F8), an antibody specific to the alternatively-spliced EDA domain of fibronectin, a marker of angiogenesis, was used [26] (Fig. 2).

\section{Reactivity of SIP(PS41) and SIP(PS72) towards phospholipids immobilized on Maxisorp}

In previous studies, the binding specificity of bavituximab, 3G4 and other anti-PS antibodies was tested in a panel of phospholipid antigens [17], whose structure is shown in Fig. 1a. We repeated similar direct ELISA experiments using anionic phospholipids (DPPS, DDPA, PI and CL) and neutral lipids (DPPE, DPPC and SM) as target antigens. SIP(PS72) exhibited selective binding to PS and phosphatidic acid, whereas SIP(PS41) also bound to cardiolipin (Fig. 3a).

\section{Flow-cytometric analysis of MDA-MB-231 cells}

The two antibodies avidly bound to MDA-MB-231 breast cancer cells in a FACS experiment, compared to the SIP(KSF) antibody [27], specific to hen egg lysozyme, which was used as negative control (Fig. 3b).

\section{Immunofluorescence on frozen tissue sections}

Immunofluorescence procedures, performed in sections of freshly frozen normal adult mouse organs and of 9 grafted tumor types (of murine and human origin), revealed that both PS72 and PS41 strongly stained the majority of the experimental tumor models, while they did not stain normal organs (Fig. 4). The F8 antibody was used as positive control, confirming the previously reported pan-tumoral specificity of this antibody [28, 26], while the antilysozyme KSF antibody stained neither tumor sections nor normal organs. In some cases (e.g., U87 tumors), 
Fig. 1 a. Chemical formulas of phospholipids that are used in the study: 1,2-dipalmitoyl-snglycero-3-phosphoserine, sodium salt (DPPS), 1,2-dipalmitoyl-snglycero-3-phosphate, sodium salt (DPPA), 1,2-dipalmitoyl-snglycero-3-phosphocholine (DPPC) and 1,2-dipalmitoyl-snglycero-3-phosphoethanolamine (DPPE), sphingomyelin (SM), cardiolipin (CL) and L-alphaphosphatidylinositol ammonium salt (PI). b. Schematic representation of an ScFv and SIP antibody format. c. Sequences of PS41 and PS72 with marked CDR regions

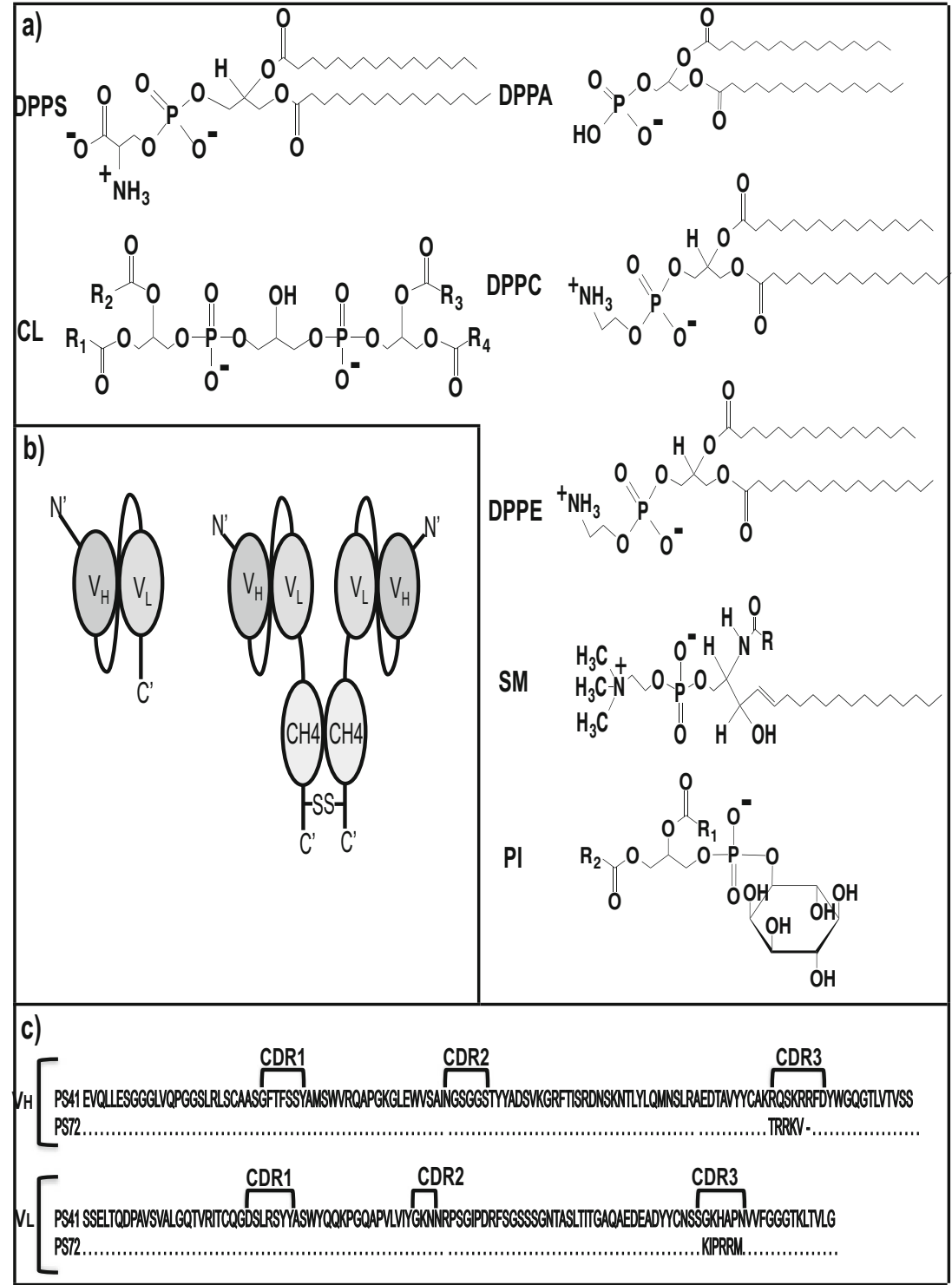

the staining patterns for PS72 and PS41 were predominantly vascular, while for other tumors (e.g., K1735, MDA-MB-231, SKRC-52, HeLa) a more diffuse staining pattern was observed.

\section{Biodistribution studies}

Radioiodinated preparations of SIP(PS41), SIP(PS72) and SIP(F8) were injected into nude mice, bearing subcutaneously-grafted SKRC-52 tumors. Twenty-four hours after intravenous administration, SIP(F8) exhibited a preferential tumor uptake $[5.2 \% \mathrm{ID} / \mathrm{g}$ in the tumor, compared to 0.51 $\% \mathrm{ID} / \mathrm{g}$ in blood], while SIP(PS41) and SIP(PS72) failed to localize to the neoplastic lesions in vivo [Supplementary Figure 2].

\section{Discussion}

We raised two fully human monoclonal antibodies, one of which bound specifically to PS and to PA (PS72), while the other antibody (PS41) also bound to CL, but neither recognized other structurally similar phospholipids, as demonstrated by ELISA and BIAcore analyses. Using freshly frozen sections of healthy murine tissues, as well as of human and murine cancers, we confirmed strong expression of PS in 8/9 tumors and almost virtual absence in healthy murine tissues. However, biodistribution studies of radioiodinated antibody preparations in tumor bearing mice failed to show preferential accumulation at the site of tumor, casting doubt at the utility of PS as a target for antibody-mediated tumor targeting.

ELISA on a panel of structurally similar phospholipids, comprising 4 anionic (DPPS, DPPA, PI and CL) and 3 neutral 


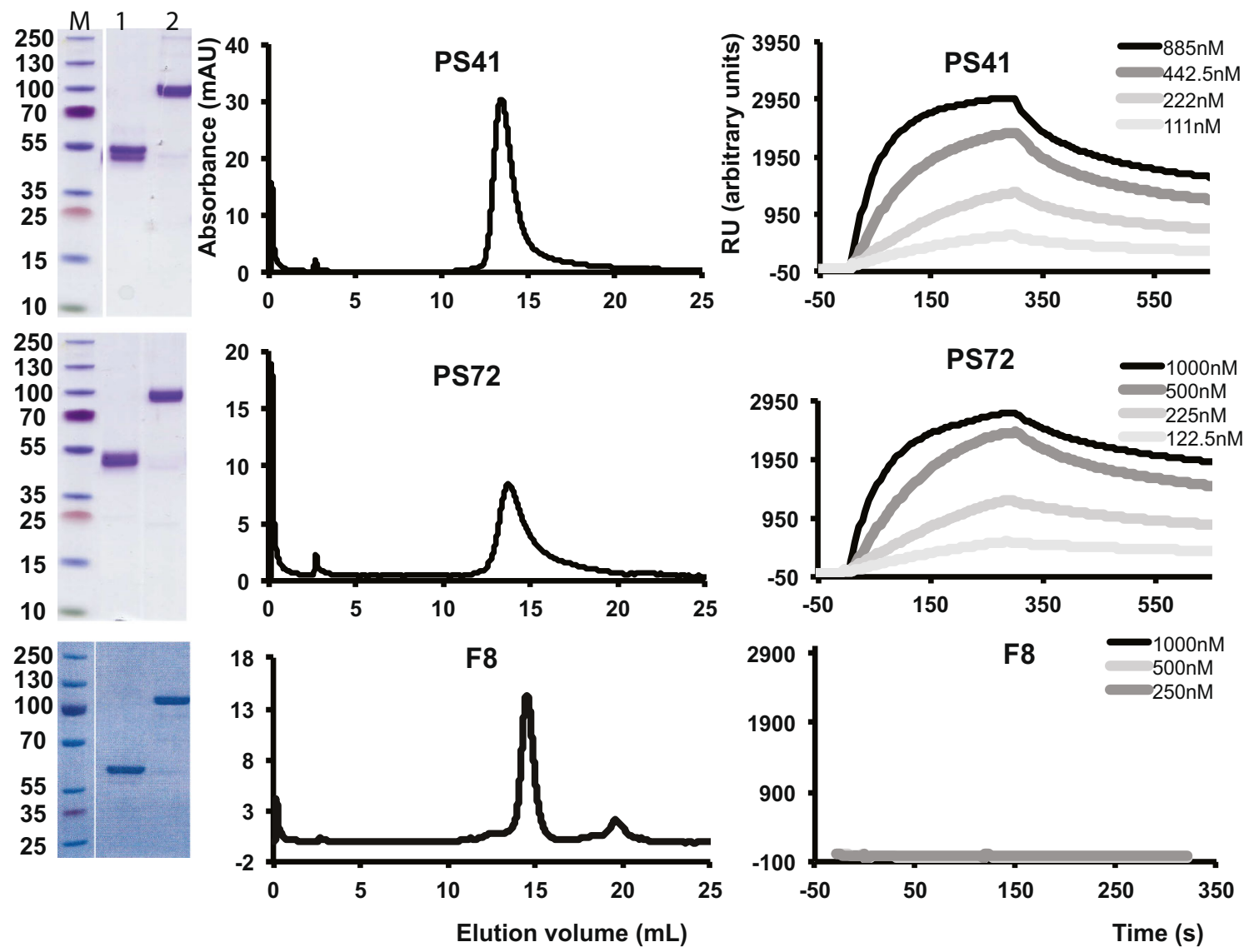

Fig. 2 SDS-PAGE profile of SIP(PS41), SIP(72) and SIP(F8) antibody fragments (left). M-marker; R - SIP under reducing conditions; NR - SIP under non-reducing conditions. Size exclusion chromatography of the

lipids (DPPE, DPPC and SM), revealed specific binding of SIP(PS72) to PS as well as to PA, while SIP(PS41) additionally bound to CL. Binding results obtained with the PS41 and PS72 antibodies suggest a higher degree of specificity compared to the bavituximab- $\beta 2 \mathrm{GP} 1$ complex, which does not discriminate between individual anionic phospholipids and binds to PS, PI, PA and CL [17]. Binding to PI, which represents $7 \%$ of plasma membrane total lipid content, may potentially contribute to off-target effects. While representing only $1 \%$ of plasma membrane's lipid content, PA blood levels lie in the nano-molar range [29], potentially requiring doseelevation of the administered antibody fragment, which recognizes both PA and PS, in order to first cover PA in the blood and reach the targeted PS in the tumor. Also, blocking of PA and its downstream metabolites like eicosanoids (e.g., prostaglandins), diacylglycerol or lyso-phosphatidic acid (LPA) may interfere with important processes such as erythrocyte aggregation, endothelial cell adhesion, thrombin generation and blood clotting [30-32]. On the other hand, specificity to CL (absent from plasma membrane) is likely to have little effect on tumor targeting [33, 34, 32].

Bavituximab was raised using hybridoma technology against endothelial cells pre-treated with an apoptosis- expressed SIP fragments on Superdex S200 column (middle). BIAcore profiles of SIP antibodies (right) against DPPS on L1 BIAcore chip (right)

inducing agent, which resulted in bavituximab being specific to a serum component ( $\beta 2 \mathrm{GP} 1)[19,17]$. In contrast to this, PS41 and PS72 were raised using antibody phage display technology against pure phosphatidylserine adsorbed on plastic surface. The ability of these antibodies to directly recognize PS was confirmed in serum-free BIAcore analysis against DPPS liposomes.

Bavituximab was studied in combination with docetaxel in a placebo-controlled double-blind clinical trial in patients with locally advanced or metastatic non-small-cell lung cancer. Vial coding discrepancies were discovered at the time of unblinding for the study and, to the best of our knowledge, the trial results have not yet been published. In previous studies, bavituximab $3 \mathrm{mg} / \mathrm{kg}$ plus docetaxel showed overall survival of 11.7 months compared to 7.3 months in the combined control arm [35].

The presence of PS on various cancer cell types had previously been reported, including those with poor treatment outcomes, e.g., glioblastoma and melanoma [4]. In addition, PS levels on the surface of tumorigenic and metastatic cell lines appear to be much higher than in normal cells $[5,4]$. In our findings, PS staining was undetectable in healthy murine organs, but an intense staining pattern was observed for various 
Fig. 3 a. Reactivity of SIP(PS41) and SIP(PS72) towards phospholipids immobilized on Maxisorp. b. Flow-cytometric analysis of MDA-MB-231 cells using SIP(PS41), SIP(PS72) and SIP(KSF)
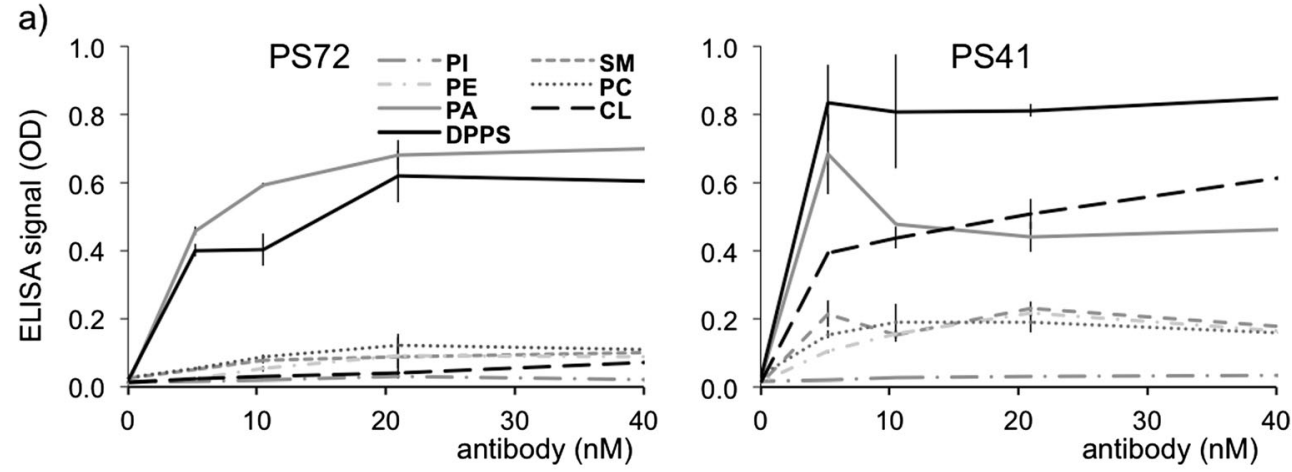

b)

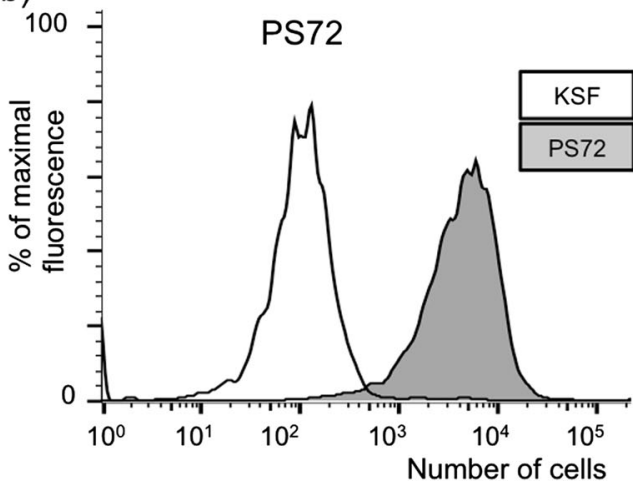

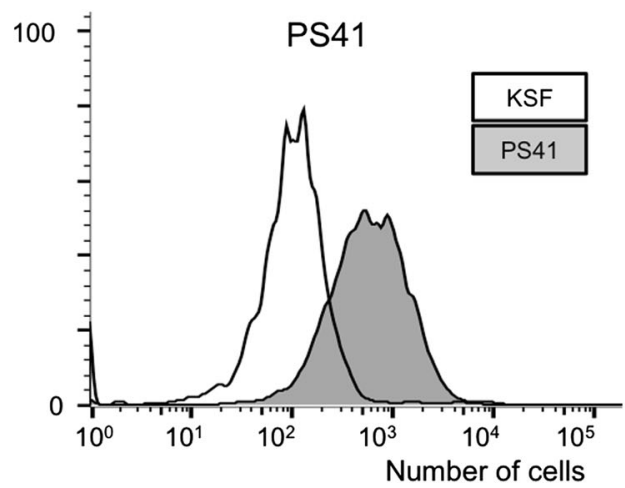

tumors (in particular, for HeLa, U87 gliomas, C1498 chloroma lesions of leukemia and K1735 melanoma). In the case of F9, RAMOS and A375, staining was not as intense and appeared to differ between SIP(PS41) and SIP(PS72).

As has been previously reported for other antibodies directed against PS [21], our antibodies failed to preferentially accumulate at the tumor site in a quantitative biodistribution study. It is not clear, at present, whether affinity-matured antibody variants may exhibit better targeting results. The antiEDA antibody F8, used as positive control in the study, had a $\mathrm{Kd}=3 \mathrm{nM}$ towards its cognate antigen [26]. In the past, Annexin $\mathrm{V}$ has often been used as an imaging agent to detect the presence of PS-positive cells in various conditions, including cardiac allograft rejection [12], acute myocardial infarction [8], unstable atherosclerotic plaque [10], rheumatoid arthritis [13], heart failure [9], dementia [11] and acute stroke [36]. Additionally, ${ }^{99 m}$ Tc-labeled Annexin $\mathrm{V}$ has been used for the imaging of chemotherapy-induced apoptosis in tumor patients [37]. Annexin V binds specifically to PS on the extracellular surface of plasma membranes in a cooperative manner and is dependent on both the phospholipid composition and $\mathrm{Ca}^{2+}$ concentration [38]. The cooperative nature of the Annexin V-PS bond is reflected in the formation of twodimensional lattices of Annexin $\mathrm{V}$ molecules upon the initial binding of the Annexin V molecule to the PS-containing binding site $[39,40]$. This structure is stabilized by protein-protein interactions, and it was demonstrated that up to 400 molecules of Annexin V could bind to PS-expressing plasma membrane even without the necessity for PS at high $\mathrm{Ca}^{2+}$ levels [41, 42]. The high-affinity binding of Annexin V to PS with a $\mathrm{Kd}$ of less than $0.1 \mathrm{nM}$ [43] may represent an important determinant for in vivo targeting applications. Other factors such as antibody degradation in vivo and increased antibody clearance, as DPL16 germline gene antibodies already demonstrated earlier [44], may also explain the observed tumor targeting performance of SIP(PS41) and SIP(PS72). At this moment in time, we do not know the reasons for the inability of the two anti-PS antibodies to preferentially localize to tumors. An insufficient binding affinity or loss of immunoreactivity upon radioiodination (not seen with other antibodies in our lab) could be possible explanations. Nonetheless, the clean staining patterns observed with PS41 and PS72 in freshly-frozen sections of normal organs, together with an intense staining of most cancer specimens tested in our analysis, reinforce the rationale for the choice of PS as an attractive (albeit difficult) target for antibody-mediated pharmacodelivery applications in those indications for which immunohistochemical studies reveal a strong antigen expression (e.g., glioblastoma, melanoma and renal cell carcinoma).

\section{Materials and methods}

1,2-dipalmitoyl-sn-glycero-3-phosphoserine, sodium salt (DPPS), 1,2-dipalmitoyl-sn-glycero-3-phosphate, 
Fig. 4 Immunofluorescence staining of murine healthy tissues, tumors xenografted in nude mice and murine tumors grafted in immunocompetent mice using SIP(KSF), SIP(PS41), SIP(PS72), SIP(F8) and anti-CD31 antibody. SIP antibodies are shown in green; they were detected using rabbit anti-human-IgE IgG antibody, which was further detected by donkey anti-rabbit IgG Alexa Fluor 488 antibody. Vessels are shown in red as the rat antimouse-CD31 antibody was detected by Alexa Fluor 594 goat anti-rat IgG antibody

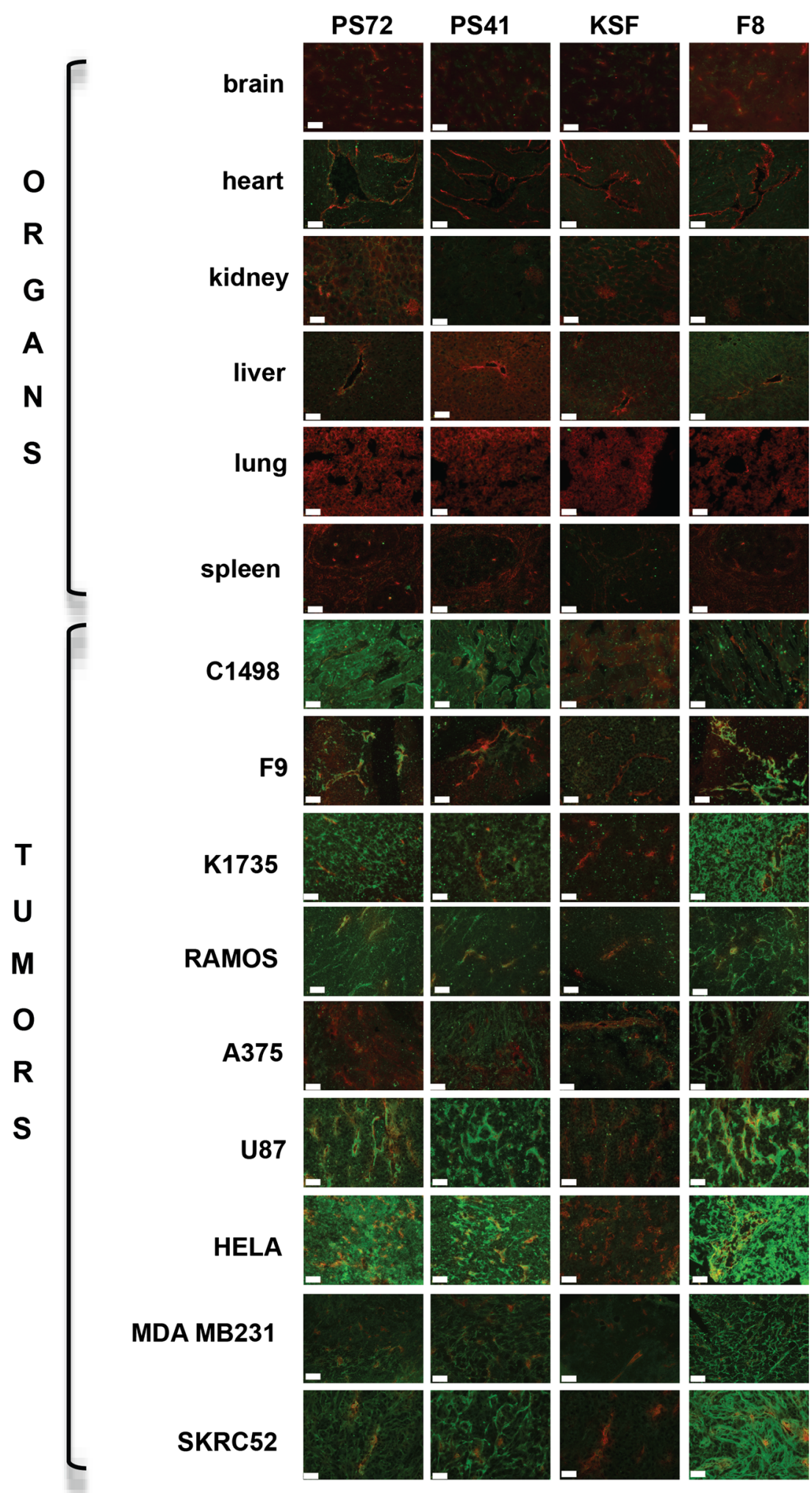

sodium salt (DPPA), 1,2-dipalmitoyl-sn-glycero-3phosphocholine (DPPC) and 1,2-dipalmitoyl-snglycero-3-phosphoethanolamine (DPPE) were purchased from Echelon Biosciences Inc. Sphingomyelin from chicken egg yolk (SM), cardiolipin solution from bovine heart (CL) and L-alpha-phosphatidylinositol ammonium salt from Glycine max (PI) were procured from Sigma. 


\section{Isolation of antibodies from phage display libraries}

DPPS was dissolved as described in manufacturer's instructions, followed by further dilution in methanol. Two hundred $\mu \mathrm{g} / \mathrm{mL}$ of the lipid solution was coated onto Maxisorp plates (Nunc). After evaporation of the solvent under reduced pressure, blocking was performed using $2 \%$ bovine serum albu$\min$ (BSA) for $3 \mathrm{~h}$. After two washing steps with phosphatebuffered saline (PBS), Philo-DIAMOND library phage preparation in $2 \% \mathrm{BSA}$ was added to the DPPS-coated-wells and incubated for $4 \mathrm{~h}$ at room temperature [23]. Unbound phage particles were washed away with 30 washings with PBS. Elution of phage particles was performed using $100 \mathrm{mM}$ solution of triethylamine. Subsequent TG1 E. coli infection and phage amplification was performed according to the established protocol [26]. Bacterial supernatants harboring soluble single-chain variable fragments $(\mathrm{scFv})$ were screened by ELISA and positive clones were further assessed for their ability to bind the immobilized antigen on a high-coatingdensity chip using surface plasmon resonance on the BIAcore3000 instrument (GE Healthcare).

\section{Characterization of scFv antibody fragments}

ScFv were expressed in TG1 E. coli and purified by affinity chromatography from the culture supernatant using Protein A Sepharose (Sino Biological) as described previously [45]. Purified scFvs were characterized by SDS-PAGE and size exclusion chromatography (SEC) on S75 Superdex column (GE Healthcare).

\section{Sequencing of scFv genes}

The DNA was amplified by PCR using primers 5'-CAG GAA ACA GCT ATG ACC ATG ATT AC-3' and 5'-GAC GTT AGT AAA TGA ATT TTC TGT ATG AGG-3' (Sigma). Sequencing was performed by GATC Biotech (Germany) according to the standard Sanger method.

\section{Cloning and expression of small immune proteins}

$\mathrm{ScFv}$ obtained from library screening were reformatted into a SIP format employing a previously described procedure [25, 24]. In brief, the $\mathrm{scFv}$ sequence was fused in frame with the sequence of the $\mathrm{CH} 4$ domain of the human $\mathrm{IgE}$ secretory isoform IgE-S2 using overlap extension PCR. The DNA fragment was inserted into pcDNA3.1 vector using HindIII-NotI restriction enzymes. The obtained plasmid was used to transiently transfect Chinese hamster ovary cells (CHO-S) as described elsewhere [46]. On Day 6 post-transfection SIPs were purified from the culture supernatant by affinity chromatography using Protein A Sepharose.

\section{BIAcore studies}

Liposome preparation of DPPS was prepared by dissolving DPPS in chloroform. Chloroform was evaporated, and the remaining lipid film was hydrated with PBS for $1 \mathrm{~h}$. Small size of liposome vesicles was achieved using a sonicator bath. Sensor chip L1 was coated with the liposome preparation of DPPS according to chip manufacturer's instructions and used for further kinetic measurements. The lipophilic sensor chip, L1, is designed for direct capture of lipid vesicles and liposomes while retaining the bilayer structure of the vesicle. Monomeric fraction of bacterially produced scFvs from the SEC analysis were isolated and used for affinity measurement using BIAcore3000 instrument as described elsewhere [47]. Kinetic measurements of SIP(PS41) and SIP(PS72) were also performed. The binding curves were analyzed with the BIA Evaluation software. As a negative control, clinical-grade F8 antibody (specific to EDA of fibronectin) was used and did not show any binding to L1 chip coated with PS.

\section{Reactivity of SIP(PS41) and SIP(PS72) towards phospholipids immobilized on Maxisorp}

Phospholipids (DPPS, DPPA, PI, CL, DPPE, DPPC and SM) were first dissolved according to manufacturer's instructions and were further dissolved in methanol. In line with the ELISA experiment performed by Ran et al. [17], $50 \mu \mathrm{g} / \mathrm{mL}$ of phospholipid solution in methanol was immobilized on Maxisorp plates (100 $\mu \mathrm{L}$ per well). Upon evaporation of the solvent under reduced pressure and blocking with $2 \%$ BSA for $2 \mathrm{~h}$, decreasing concentrations of SIP(PS41) and $\mathrm{SIP}(\mathrm{PS} 72)$ in $2 \% \mathrm{BSA}$ were incubated for $2 \mathrm{~h}$, followed by subsequent detection with rabbit anti-human-IgE-IgG (diluted 1:200 in $2 \% \mathrm{BSA}$, incubated for $2 \mathrm{~h}$ ) and anti-rabbit-IgG-HRP (diluted 1:1000 in $2 \% \mathrm{BSA}$, incubated for $1 \mathrm{~h}$ ). Rinsing with PBS was performed in between all incubation steps. All measurements were performed in triplicates.

\section{Flow-cytometric analysis of MDA-MB-231 cells}

MDA-MB-231 cells were cultivated in DMEM medium with $10 \%$ FCS. The cells were detached using Acutase solution (Sigma-Aldrich). The staining was performed with SIP(PS41) and $\operatorname{SIP}(72)$, which were detected with rabbit anti-human-IgE $\operatorname{IgG}$ (Dako), followed by Alexa Fluor 594 goat anti-rabbit IgG antibody (Molecular Probes). The analysis was performed using FACS Canto II (BD Biosciences) and the FlowJo 8.7 software.

\section{Immunofluorescence on frozen tissue sections}

Healthy tissues were excised from C57BL/6 mice while human tumor xenografts were grown subcutaneously and 
excised from BALB/c nude mice. Murine tumors were grown and excised from appropriate mouse strains. K1735 was grown in C3H mice, F9 in Sv129, while C1498 was grown in C57BL/6. We harvested selected tissues immediately upon the animal was sacrificed, and tissues were embedded in freezing medium (Microm) and stored at $-80{ }^{\circ} \mathrm{C}$ until sectioned. Tissue sections $(10 \mu \mathrm{m})$ were fixed for $10 \mathrm{~min}$ with ice-cold acetone, rehydrated with PBS and blocked with $20 \%$ FCS in PBS. Purified SIP (PS41) and (PS72) were applied to slices at a concentration of $2 \mu \mathrm{g} / \mathrm{mL}$ in $3 \%$ bovine serum albumin and incubated for $2 \mathrm{~h}$. The $\mathrm{CH} 4$ domain of SIPs was detected with rabbit anti-human-IgE IgG (Dako) (diluted 1:200, incubated for $2 \mathrm{~h}$ ), which was then detected by Alexa Fluor 488 goat anti-rabbit IgG antibody (diluted 1:1000, incubated for $1 \mathrm{~h}$ ) (Molecular Probes). Blood vessels were detected with rat antimouse-CD31 antibody (diluted 1:200, incubated for $2 \mathrm{~h}$ ) (Invitrogen) followed by donkey Alexa Fluor 594 anti-rat IgG antibody (diluted 1:1000, incubated for $1 \mathrm{~h}$ ) (Molecular Probes). Nuclei were counterstained with DAPI (diluted 1.5000 , incubated for $1 \mathrm{~h}$ ) (Invitrogen). All commercial binding reagents were diluted according to the manufacturer's recommendation in $3 \%$ BSA solution. Rinsing with PBS was performed in between all incubation steps. Slides were mounted with fluorescent mounting medium (Dako) and analyzed with a Zeiss AxioVision 4.7 image analysis software (Carl Zeiss AG).

\section{Biodistribution studies}

The in vivo targeting performance of SIP fragments was evaluated by a quantitative biodistribution analysis as previously described [48]. Briefly, purified antibody preparations were radiolabeled with ${ }^{125} \mathrm{I}$ using the Iodogen method [49, 50] and injected into the tail vein of BALB/c nude mice bearing subcutaneously implanted SKRC-52 tumor (about $10 \mu \mathrm{g}$ per mouse). Mice were sacrificed $24 \mathrm{~h}$ after injection. Organs were weighed and radioactivity was counted with a PackardCobra $\gamma$ counter. Radioactivity content of representative organs was expressed as the percentage of the injected dose per gram of tissue $(\% \mathrm{ID} / \mathrm{g})$.

\begin{abstract}
Acknowledgments Financial support from ETH Zürich, the Swiss National Science Foundation, the Kommission für Technologie und Innovation (KTI MedTech Award) and the European Union (PRIAT FP7 Project) is gratefully acknowledged. Also, we would like to thank Dr. Sarah Wulhfard and Dr. Doriana Triggiani for their help with the biodistribution experiment.
\end{abstract}

Conflict of interest The authors declare that they have no conflict of interest.

\section{References}

1. Fadok VA, de Cathelineau A, Daleke DL, Henson PM, Bratton DL (2001) Loss of phospholipid asymmetry and surface exposure of phosphatidylserine is required for phagocytosis of apoptotic cells by macrophages and fibroblasts. J Biol Chem 276(2):1071-1077. doi:10.1074/jbc.M003649200

2. Fischer K, Voelkl S, Berger J, Andreesen R, Pomorski T, Mackensen A (2006) Antigen recognition induces phosphatidylserine exposure on the cell surface of human CD8+ T cells. Blood 108(13):4094-4101

3. Ran S, Thorpe PE (2002) Phosphatidylserine is a marker of tumor vasculature and a potential target for cancer imaging and therapy. Int J Radiat Oncol 54(5):1479-1484. doi:10.1016/s0360-3016(02) 03928-7

4. Riedl S, Rinner B, Asslaber M, Schaider H, Walzer S, Novak A, Lohner K, Zweytick D (2011) In search of a novel target phosphatidylserine exposed by non-apoptotic tumor cells and metastases of malignancies with poor treatment efficacy. BBA Biomembr 1808(11):2638-2645. doi:10.1016/j.bbamem.2011.07. 026

5. Utsugi T, Schroit AJ, Connor J, Bucana CD, Fidler IJ (1991) Elevated expression of phosphatidylserine in the outer membrane leaflet of human tumor cells and recognition by activated human blood monocytes. Cancer Res 51(11):3062-3066

6. Yagle KJ, Eary JF, Tait JF, Grierson JR, Link JM, Lewellen B, Gibson DF, Krohn KA (2005) Evaluation of 18F-annexin V as a PET imaging agent in an animal model of apoptosis. J Nucl Med 46(4):658-666

7. Zhao D, Stafford JH, Zhou H, Thorpe PE (2011) Near-infrared optical imaging of exposed phosphatidylserine in a mouse glioma model. Transl Oncol 4(6):355-364

8. Hofstra L, Liem IH, Dumont EA, Boersma HH, van Heerde WL, Doevendans PA, DeMuinck E, Wellens HJJ, Kemerink GJ, Reutelingsperger CPM, Heidendal GA (2000) Visualisation of cell death in vivo in patients with acute myocardial infarction. Lancet 356(9225):209-212. doi:10.1016/S0140-6736(00)02482-X

9. Kietselaer BLJH, Reutelingsperger CPM, Boersma HH, Heidendal GAK, Liem IH, Crijns HJGM, Narula J, Hofstra L (2007) Noninvasive detection of programmed cell loss with $99 \mathrm{mTc}-$ labeled annexin A5 in heart failure. J Nucl Med 48(4):562-567. doi:10.2967/jnumed.106.039453

10. Kolodgie FD, Petrov A, Virmani R, Narula N, Verjans JW, Weber DK, Hartung D, Steinmetz N, Vanderheyden JL, Vannan MA, Gold HK, Reutelingsperger CPM, Hofstra L, Narula J (2003) Targeting of apoptotic macrophages and experimental atheroma with radiolabeled annexin V: a technique with potential for noninvasive imaging of vulnerable plaque. Circulation 108(25):3134-3139. doi: 10.1161/01.cir.0000105761.00573.50

11. Lampl Y, Lorberboym M, Blankenberg FG, Sadeh M, Gilad R (2006) Annexin V SPECT imaging of phosphatidylserine expression in patients with dementia. Neurology 66(8):1253-1254. doi: 10.1212/01.wnl.0000208436.75615.8c

12. Narula J, Acio ER, Narula N, Samuels LE, Fyfe B, Wood D, Fitzpatrick JM, Raghunath PN, Tomaszewski JE, Kelly C, Steinmetz N, Green A, Tait JF, Leppo J, Blankenberg FG, Jain D, Strauss HW (2001) Annexin-V imaging for noninvasive detection of cardiac allograft rejection. Nat Med 7(12):1347-1352

13. Post AM, Katsikis PD, Tait JF, Geaghan SM, Strauss HW, Blankenberg FG (2002) Imaging cell death with radiolabeled annexin $\mathrm{V}$ in an experimental model of rheumatoid arthritis. $\mathrm{J}$ Nucl Med 43(10):1359-1365

14. Bondanza A, Zimmermann VS, Rovere-Querini P, Turnay J, Dumitriu IE, Stach CM, Voll RE, Gaipl US, Bertling W, Pöschl E, Kalden JR, Manfredi AA, Herrmann M (2004) Inhibition of 
phosphatidylserine recognition heightens the immunogenicity of irradiated lymphoma cells in vivo. J Exp Med 200(9):1157-1165. doi:10.1084/jem.20040327

15. He J, Yin Y, Luster TA, Watkins L, Thorpe PE (2009) Antiphosphatidylserine antibody combined with irradiation damages tumor blood vessels and induces tumor immunity in a rat model of glioblastoma. Clin Cancer Res 15(22):6871-6880. doi: 10.1158/1078-0432.ccr-09-1499

16. Huang X, Bennett M, Thorpe PE (2005) A monoclonal antibody that binds anionic phospholipids on tumor blood vessels enhances the antitumor effect of docetaxel on human breast tumors in mice. Cancer Res 65(10):4408-4416. doi:10.1158/0008-5472.can-050031

17. Ran S, He J, Huang X, Soares M, Scothorn D, Thorpe PE (2005) Antitumor effects of a monoclonal antibody that binds anionic phospholipids on the surface of tumor blood vessels in mice. Clin Cancer Res 11(4):1551-1562. doi:10.1158/1078-0432.ccr-04-1645

18. Beck AW, Luster TA, Miller AF, Holloway SE, Conner CR, Barnett CC, Thorpe PE, Fleming JB, Brekken RA (2006) Combination of a monoclonal anti-phosphatidylserine antibody with gemcitabine strongly inhibits the growth and metastasis of orthotopic pancreatic tumors in mice. Int J Cancer 118(10):2639-2643. doi:10.1002/ijc. 21684

19. Luster TA, He J, Huang X, Maiti SN, Schroit AJ, de Groot PG, Thorpe PE (2006) Plasma protein $\beta$-2-glycoprotein 1 mediates interaction between the anti-tumor monoclonal antibody $3 \mathrm{G} 4$ and anionic phospholipids on endothelial cells. J Biol Chem 281(40): 29863-29871. doi:10.1074/jbc.M605252200

20. Tomasi M, Hiromasa Y, Pope MR, Gudlur S, Tomich JM, Fleming SD (2012) Human $\beta 2$-glycoprotein I attenuates mouse intestinal ischemia/reperfusion induced injury and inflammation. Mol Immunol 52(3-4):207-216. doi:10.1016/j.molimm.2012.05.018

21. Stafford JH, Hao G, Best AM, Sun X, Thorpe PE (2013) Highly specific PET imaging of prostate tumors in mice with an iodine124-labeled antibody fragment that targets phosphatidylserine. PLoS ONE 8(12), e84864. doi:10.1371/journal.pone.0084864

22. Wahl RL, Parker CW, Philpott GW (1983) Improved radioimaging and tumor localization with monoclonal F(ab')2. J Nucl Med 24(4): 316-325

23. Weber M, Bujak E, Putelli A, Villa A, Matasci M, Gualandi L, Hemmerle T, Wulhfard S, Neri D (2014) A highly functional synthetic phage display library containing over 40 billion human antibody clones. PLoS ONE 9(6), e100000. doi:10.1371/journal.pone. 0100000

24. Borsi L, Balza E, Bestagno M, Castellani P, Carnemolla B, Biro A, Leprini A, Sepulveda J, Burrone O, Neri D, Zardi L (2002) Selective targeting of tumoral vasculature: comparison of different formats of an antibody (L19) to the ED-B domain of fibronectin. Int J Cancer 102:75-85

25. Zuberbühler K, Palumbo A, Bacci C, Giovannoni L, Sommavilla R, Kaspar M, Trachsel E, Neri D (2009) A general method for the selection of high-level scFv and IgG antibody expression by stably transfected mammalian cells. Protein Eng Des Sel 22(3):169-174. doi:10.1093/protein/gzn068

26. Villa A, Trachsel E, Kaspar M, Schliemann C, Sommavilla R, Rybak J, Rosli C, Borsi L, Neri D (2008) A high-affinity human monoclonal antibody specific to the alternatively spliced EDA domain of fibronectin efficiently targets tumor neo-vasculature in vivo. Int J Cancer 122:2405-2413

27. Frey K, Zivanovic A, Schwager K, Neri D (2011) Antibody-based targeting of interferon-alpha to the tumor neovasculature: a critical evaluation. Integr Biol 3(4):468-478. doi:10.1039/c0ib00099j

28. Rybak J-N, Roesli C, Kaspar M, Villa A, Neri D (2007) The extradomain a of fibronectin is a vascular marker of solid tumors and metastases. Cancer Res 67(22):10948-10957. doi:10.1158/00085472.can-07-1436
29. Purpura M, Jager R, Joy J, Lowery R, Moore J, Wilson J (2013) Effect of oral administration of soy-derived phosphatidic acid on concentrations of phosphatidic acid and lyso-phosphatidic acid molecular species in human plasma. Int J Sport Nutr 10(Suppl 1):P22

30. Mills GB, Moolenaar WH (2003) The emerging role of lysophosphatidic acid in cancer. Nat Rev Cancer 3(8):582-591

31. Noh J-Y, Lim K-M, Bae O-N, Chung S-M, Lee S-W, Joo K-M, Lee S-D, Chung J-H (2010) Procoagulant and prothrombotic activation of human erythrocytes by phosphatidic acid, vol 299. vol 2. doi:10. 1152/ajpheart.01144.2009

32. Leventis PA, Grinstein S (2010) The distribution and function of phosphatidylserine in cellular membranes. Annu Rev Biophys 39(1):407-427. doi:10.1146/annurev.biophys.093008.131234

33. Fridriksson EK, Shipkova PA, Sheets ED, Holowka D, Baird B, McLafferty FW (1999) Quantitative analysis of phospholipids in functionally important membrane domains from RBL-2H3 mast cells using tandem high-resolution mass spectrometry $\dagger$. Biochemistry 38(25):8056-8063. doi:10.1021/bi9828324

34. Bevers E, Comfurius P, Zwaal R (1996) Regulatory mechanisms in maintenance and modulation of transmembrane lipid asymmetry: pathophysiological implications. Lupus 5(5):480-487. doi:10. 1177/096120339600500531

35. Peregrine (2014) Data presented at ASCO shows promising 11.7 month median overall survival in second- line NSCLC patients treated with Peregrine Pharmaceuticals' novel immunotherapy bavituximab. Accessed 27 June 2014

36. Lorberboym M, Blankenberg FG, Sadeh M, Lampl Y (2006) In vivo imaging of apoptosis in patients with acute stroke: correlation with blood-brain barrier permeability. Brain Res 1103(1):1319. doi:10.1016/j.brainres.2006.05.073

37. Belhocine T, Steinmetz N, Hustinx R, Bartsch P, Jerusalem G, Seidel L, Rigo P, Green A (2002) Increased uptake of the apoptosis-imaging agent $99 \mathrm{mTc}$ recombinant human annexin $\mathrm{V}$ in human tumors after one course of chemotherapy as a predictor of tumor response and patient prognosis. Clin Cancer Res 8(9):27662774

38. Schlaepfer DD, Haigler HT (1987) Characterization of Ca2+ -dependent phospholipid binding and phosphorylation of lipocortin I. J Biol Chem 262(14):6931-6937

39. Andree HA, Reutelingsperger CP, Hauptmann R, Hemker HC, Hermens WT, Willems GM (1990) Binding of vascular anticoagulant alpha (VAC alpha) to planar phospholipid bilayers. J Biol Chem 265(9):4923-4928

40. Tait JF, Gibson D (1992) Phospholipid binding of annexin V: effects of calcium and membrane phosphatidylserine content. Arch Biochem Biophys 298(1):187-191. doi:10.1016/0003-9861(92) 90111-9

41. Andree HA, Stuart MC, Hermens WT, Reutelingsperger CP, Hemker HC, Frederik PM, Willems GM (1992) Clustering of lipid-bound annexin $\mathrm{V}$ may explain its anticoagulant effect. J Biol Chem 267(25):17907-17912

42. Mosser G, Ravanat C, Freyssinet J-M, Brisson A (1991) Subdomain structure of lipid-bound annexin-V resolved by electron image analysis. J Mol Biol 217(2):241-245. doi:10.1016/00222836(91)90538-H

43. Tait JF, Gibson D, Fujikawa K (1989) Phospholipid binding properties of human placental anticoagulant protein-I, a member of the lipocortin family. J Biol Chem 264(14):7944-7949

44. Pfaffen S, Frey K, Stutz I, Roesli C, Neri D (2010) Tumourtargeting properties of antibodies specific to MMP-1A, MMP-2 and MMP-3. Eur J Nucl Med Mol Imaging 37(8):1559-1565. doi:10.1007/s00259-010-1446-9

45. Silacci M, Brack S, Schirru G, Marlind J, Ettorre A, Merlo A, Viti F, Neri D (2005) Design, construction, and characterization of a large synthetic human antibody phage display library. Proteomics 5: 2340-2350 
46. List T, Neri D (2012) Biodistribution studies with tumor-targeting bispecific antibodies reveal selective accumulation at the tumor site. mAbs 4(6):775-783

47. Silacci M, Brack S, Spath N, Buck A, Hillinger S, Arni S, Weder W, Zardi L, Neri D (2006) Human monoclonal antibodies to domain C of tenascin-C selectively target solid tumors in vivo. Protein Eng Des Sel 19:471-478

48. Tarli L, Balza E, Viti F, Borsi L, Castellani P, Berndorff D, Dinkelborg L, Neri D, Zardi L (1999) A high-affinity human antibody that targets tumoral blood vessels. Blood 94(1):192-198
49. Fraker PJ (2012) Reprint of "Protein and cell membrane iodinations with a sparingly soluble chloroamide, 1,3,4,6Tetrachloro-3a,6a-Diphenylglycoluril". Biochem Biophys Res Commun 425(3):510-518. doi:10.1016/j.bbrc.2012. 08.017

50. Salacinski PRP, McLean C, Sykes JEC, Clement-Jones VV, Lowry PJ (1981) Iodination of proteins, glycoproteins, and peptides using a solid-phase oxidizing agent, 1,3,4,6-tetrachloro-3 $\alpha, 6 \alpha$-diphenyl glycoluril (Iodogen). Anal Biochem 117(1):136-146. doi:10.1016/ 0003-2697(81)90703-X 